Роман С. Торшин, Георгий С. Сорокоумов, Дмитрий В. Бобровский, Александр А. Демидов, Анастасия В. Уланова

ИЗМЕРЕНИЕ КРИТЕРИАЛЬНЫХ ПАРАМЕТРОВ АНАЛОГО-ЦИФРОВЫХ

ПРЕОБРАЗОВАТЕЛЕЙ И КОНТРОЛЬ ИХ ИЗМЕНЕНИЯ ВО ВРЕМЯ РАДИАЦИОННОГО ЭКСПЕРИМЕНТА

\author{
Роман С. Торшин ${ }^{1}$, Георгий С. Сорокоумов ${ }^{2}$, Дмитрий В. Бобровский ${ }^{3}$, \\ Александр А. Демидов ${ }^{4}$, Анастасия В. Уланова ${ }^{5}$ \\ ${ }^{1-5}$ Акиионерное общество «Экспериментальное научно-производственное объединение \\ СПЕЦИАЛИЗИРОВАННЫЕ ЭЛЕКТРОННЫЕ СИСТЕМЫ», \\ Каширское ш., 31, Москва, 115409, Россия \\ le-mail: rstor@spels.ru,https://orcid.org/0000-0001-8053-1343 \\ 2e-mail: gssor@spels.ru,https://orcid.org/0000-0002-8446-6144 \\ 3e-mail:dvbob@spels.ru,https://orcid.org/0000-0003-3036-2953 \\ 4e-mail: aadem@spels.ru,https://orcid.org/0000-0001-7123-7644 \\ 5e-mail: avulan@spels.ru,https://orcid.org/0000-0001-7376-6339

\section{ИЗМЕРЕНИЕ КРИТЕРИАЛЬНЫХ ПАРАМЕТРОВ АНАЛОГО-ЦИФРОВЫХ ПРЕОБРАЗОВАТЕЛЕЙ И КОНТРОЛЬ ИХ ИЗМЕНЕНИЯ ВО ВРЕМЯ РАДИАЦИОННОГО ЭКСПЕРИМЕНТА DOI: http://dx.doi.org/10.26583/bit.2020.3.07}

Аннотация. В данной работе апробируется метод тестирования микросхем аналого-цифровых преобразователей (АЦП), предназначенный для измерения основных параметров преобразователей методом гистограмм (или плотности кода). Данный метод хорошо подходит для современных высокочастотных АЦП с широкой полосой, совместим со стандартным программным обеспечением персональных компьютеров (ПК) и оценочных плат производителей АЦП, дает возможность на том же оборудовании определять и динамические характеристики АЦП, используя методы цифровой обработки сигнала (ЦОС). В качестве контрольно-измерительного оборудования, необходимого для проведения теста, использовались модульные измерительные приборы фирмы National Instruments, а также генератор сигналов SMA100B фирмы ROHDE\&SCHWARZ. C помощью данного метода вычислены основные статические и динамические параметры АЦП AD7888. Приведена деградация критериальных параметров АЦП AD7888 при воздействии поглощенной дозы на рентгеновском источнике «РИК-0401».

Ключевые слова: аналого-иифровой преобразователь, метод гистограмм, статические параметры АЦП, динамические параметры АЦП, тестирование микросхем, поглощенная доза. Для иитирования.: ТОРШИН, Роман С. et al. ИЗМЕРЕНИЕ КРИТЕРИАЛЬНЫХ ПАРАМЕТРОВ АНАЛОГОЦИФРОВЫХ ПРЕОБРАЗОВАТЕЛЕЙ И КОНТРОЛЬ ИХ ИЗМЕНЕНИЯ ВО ВРЕМЯ РАДИАЦИОННОГО ЭКСПЕРИМЕНТА.Безопасность информаиионных технологий, [S.l.], v. 27, n. 3, p. 76-88, 2020. ISSN 20747136. Доступно на: <https://bit.mephi.ru/index.php/bit/article/view/1294>. Дата достуna: 08 sep. 2020. DOI: http://dx.doi.org/10.26583/bit.2020.3.07.

$$
\begin{aligned}
& \text { Roman S. Torshin }{ }^{1} \text {, Georgy S. Sorokoumov }{ }^{2} \text {, Dmitry V. Bobrovsky }{ }^{3} \text {, } \\
& \text { Alexander A. Demidov }{ }^{4} \text {, Anastasya V. Ulanova }{ }^{5} \\
& { }^{1-5} \text { Joint Stock Company "Experimental Research and Production Association } \\
& \text { SPECIAL ELECTRONIC SYSTEM" } \\
& \text { Kashirskoe sh., 31, Moscow, 115409, Russia } \\
& \text { 1e-mail: rstor@spels.ru,https://orcid.org/0000-0001-8053-1343 } \\
& \text { 2e-mail: gssor@spels.ru,https://orcid.org/0000-0002-8446-6144 } \\
& \text { 3e-mail:dvbob@spels.ru,https://orcid.org/0000-0003-3036-2953 } \\
& { }^{4} \text { e-mail: aadem@spels.ru,https://orcid.org/0000-0001-7123-7644 } \\
& \text { se-mail: avulan@spels.ru,https://orcid.org/0000-0001-7376-6339 }
\end{aligned}
$$

\title{
Measurement of criteria parameters of analog-to-digital converters and monitoring of their changes during the radiation experiment DOI: http://dx.doi.org/10.26583/bit.2020.3.07
}


Роман С. Торшин, Георгий С. Сорокоумов, Дмитрий В. Бобровский,

Александр А. Демидов, Анастасия В. Уланова

ИЗМЕРЕНИЕ КРИТЕРИАЛЬНЫХ ПАРАМЕТРОВ АНАЛОГО-ЦИФРОВЫХ

ПРЕОБРАЗОВАТЕЛЕЙ И КОНТРОЛЬ ИХ ИЗМЕНЕНИЯ ВО ВРЕМЯ

РАДИАЦИОННОГО ЭКСПЕРИМЕНТА

Abstract. The paper presents a method for testing ADC, intended for measurement the basic parameters of converters by histogram test (or code density test). This method is ideally suited for modern wide bandwidth high precision ADCs and is universally accepted, especially with the proliferation of standard PC-based software and ADC manufacturer's evaluation boards. The histogram and Fast Fourier transform (FFT) tests use essentially the same hardware, both are normally part of a comprehensive ADC test plan [1]. Modular Instrumentation System PXI of NI and Signal Generator SMA100B (ROHDE \& SCHWARZ) were used as test equipment for the tests. The basic static and dynamic parameters of the ADC AD7888 were calculated using this method. The degradation of the main parameters of the ADC AD7888 under the influence of the absorbed dose at the «RIK-0401» X-ray unit is presented in the paper.

Keywords: analog to digital converter, histogram test, dynamic parameters ADC, static parameters ADC, testing microchips, absorbed dose.

For citation: TORSHIN, Roman S. et al. Measurement of criteria parameters of analog-to-digital converters and monitoring of their changes during the radiation experiment. IT Security (Russia), [S.l.], v. 27, n. 3, p. 76-88, 2020. ISSN 2074-7136. Available at: <https://bit.mephi.ru/index.php/bit/article/view/1294>. Date accessed: 08 sep. 2020. DOI: http://dx.doi.org/10.26583/bit.2020.3.07.

\section{Введение}

Современный мир оперирует огромными объемами информации, передача и обработка которых проводится с помощью автоматических и автоматизированных систем. Большинство первичных блоков систем, осуществляющих прием информации, оснащены трактами предварительной обработки аналогового сигнала и перевода его в цифровой вид. Одной из областей применения автоматических систем является аппаратура космического назначения, для которой критичным являются вопросы безотказной работы и сроки активного существования аппаратуры. Таким образом, вопросы безопасности информации неразрывно связаны с исследованием параметров стойкости микросхем АЦП при воздействии ионизирующего излучения космического пространства.

Существует множество методов для тестирования статических и динамических характеристик АЦП, но тесты на базе ЦОС стали практически универсальными промышленными стандартами для современных преобразователей. В статье предлагается гистограммный метод тестирования АЦП, который заключается в накоплении большого количества оцифрованных отсчетов входного сигнала с известной плотностью вероятности за определенный период времени.

\section{1. Описание метода гистограмм для тестирования статических параметров АЦП}

Характеристика преобразования (ХП) АЦП определяется при помощи статистического анализа отсчетов. Входным сигналом для гистограммного метода является линейно изменяющийся, или синусоидальный сигнал, слегка выходящий за пределы входного диапазона АЦП. В ходе теста подсчитывается количество выпадений каждого кода. Конфигурация гистограммного тестера представлена на рис. 1. Частота входного сигнала не должна быть субгармоникой частоты дискретизации АЦП, в противном случае кодовые комбинации в каждом периоде входного сигнала будут повторяться, что приведет к искажению измерения дифференциальной нелинейности (ДНЛ) и сделает их бессмысленными [1]. 
Роман С. Торшин, Георгий С. Сорокоумов, Дмитрий В. Бобровский,

Александр А. Демидов, Анастасия В. Уланова

ИЗМЕРЕНИЕ КРИТЕРИАЛЬНЫХ ПАРАМЕТРОВ АНАЛОГО-ЦИФРОВЫХ

ПРЕОБРАЗОВАТЕЛЕЙ И КОНТРОЛЬ ИХ ИЗМЕНЕНИЯ ВО ВРЕМЯ

РАДИАЦИОННОГО ЭКСПЕРИМЕНТА

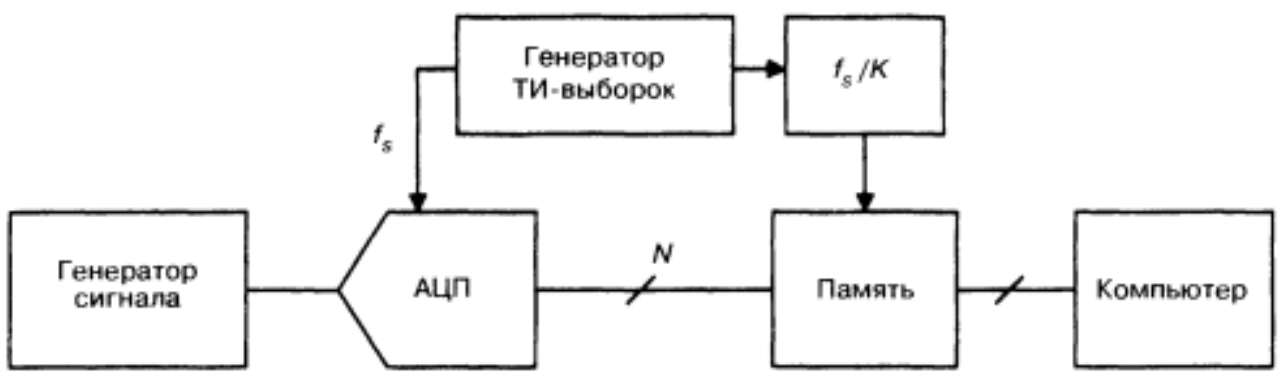

Рис. 1. Структурная схема гистограммного метода

(Fig. 1. Flow chart of the histogram test)

Измерение передаточной характеристики АЦП, по сути, направлено на определение уровней напряжения, соответствующих межкодовым переходам. На рис. 2а представлена статическая передаточная характеристика, которая задана через кодовые переходы, которые могут быть измерены напрямую. Сложность определения уровня межкодового перехода заключается в том, что код АЦП даже при фиксированном значении входного напряжения, при приближении уровня входного сигнала к уровню межкодового перехода, может интенсивно переключаться, что особенно характерно для быстродействующих преобразователей (рис. 2б) [2, 3].

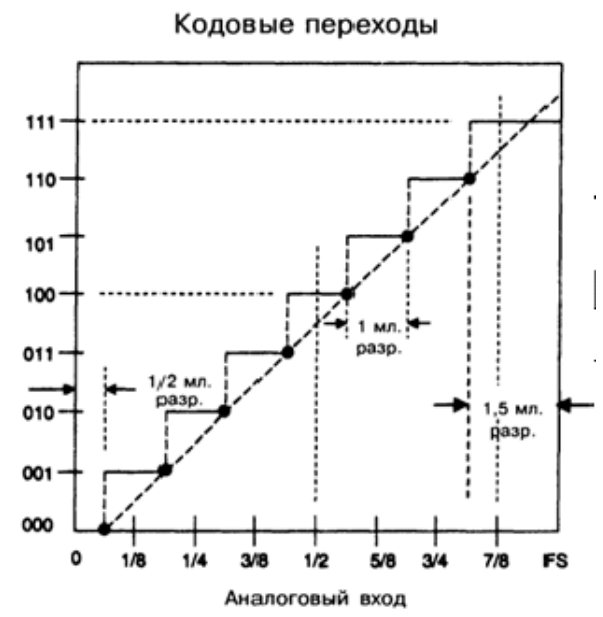

Pис. 2a. Статическая ХП АЦП

(Fig. 2a. ADC static transfer function)

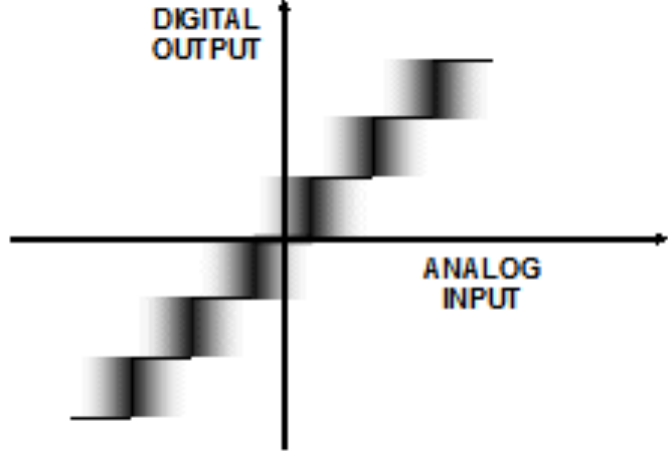

Рис. 26. Кодовые переходы реальной ХП АЦП

(Fig. 2b. Code transitions of the real ADC transfer function)

Суть гистограммного метода тестирования АЦП заключается в том, что для кодов от 0 до $2^{\mathrm{N}-1}$ собирается статистика их выпадения, подсчитывается весовой коэффициент, соответствующий частоте выпадения каждого кода (или реальный шаг квантования). В случае линейного изменения тестового сигнала идеальный шаг квантования можно вычислить следующим образом:

$$
v_{i}=\frac{V_{r e f}}{2^{N}} .
$$

Реальный шаг квантования определяется согласно формуле:

$$
v_{j}=\frac{m_{j} * \Delta \mathrm{V}}{M}
$$

где $\Delta V$ - диапазон изменения входного аналогового сигнала, $m_{j}-$ количество событий $j-$ го кода, $\mathrm{M}=\sum_{j=0}^{2^{N}-1} m_{j}-$ полное количество событий всех кодов. 
Роман С. Торшин, Георгий С. Сорокоумов, Дмитрий В. Бобровский,

Необходимо учитывать число отсчетов переполнения, соответствующих попаданиям в интервалы кодов из одних нулей (код 0) и одних единиц (код $2^{\mathrm{N}-1}$ ). Сигнал необходимо отрегулировать таким образом, чтобы АЦП не был сильно перегружен (рекомендуется работа с 10 \%-ой перегрузкой) $[4,5]$.

Типичный вид гистограммы представлен на рис. 3, на котором легко определить широкие, узкие и пропущенные коды.

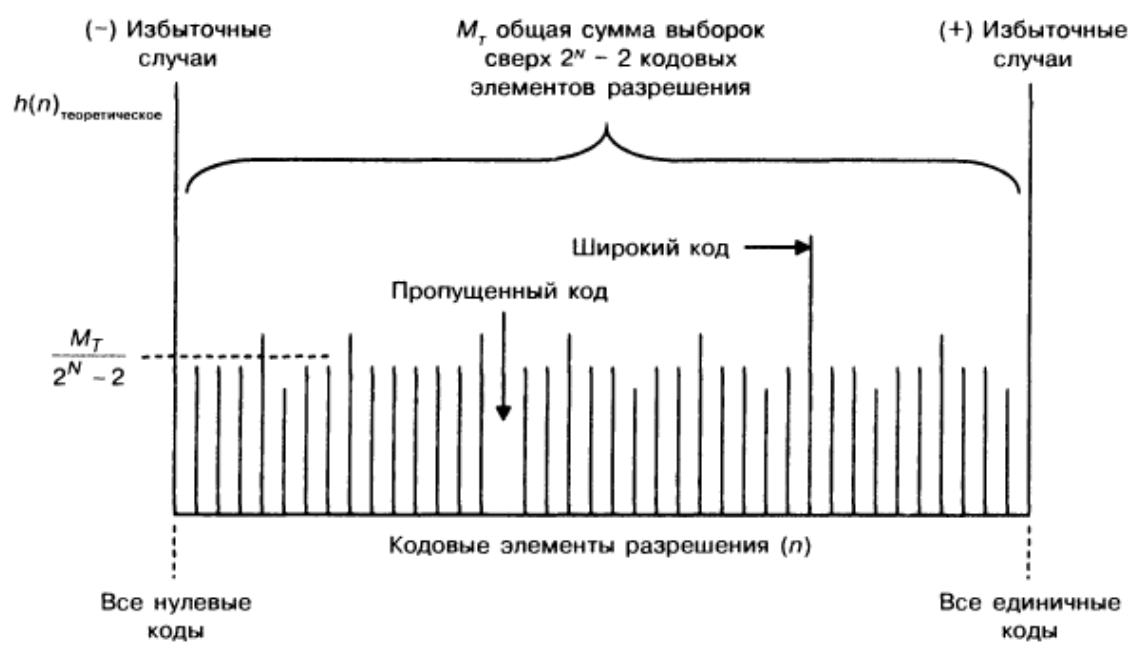

Рис. 3. Типичный вид гистограммы для линейно возрастающего входного сигнала (Fig. 3. Typical histogram for a linear input ramp signal)

Дифференциальная нелинейность (ДНЛ) определяется выражением:

$$
D N L_{j}=\frac{\left(v_{j}-v_{i}\right)}{v_{i}}
$$

После построения аппроксимирующей прямой вида «ax+b» определяется интегральная нелинейность (ИНЛ):

$$
I N L_{j}=\frac{\frac{n_{j}-b}{a}-\left(V_{0}+\Delta \mathrm{V} * \frac{\frac{m_{j}}{2}+\sum_{k=0}^{k-1} m_{k}}{M}\right)}{v_{i}},
$$

где $n_{j}$ - текущее значение кода.

Погрешность смещения нуля (аддитивная погрешность) может быть определена как смещение всей ХП АЦП влево или вправо относительно идеальной ХП и определяется согласно формуле:

$$
E_{0}=\frac{\left(v_{i} / 2-\left(V_{0}+\frac{m_{0} * \Delta V}{M}\right)\right)}{v_{i}}
$$

где $V_{0}$-начальное значение входного тестового напряжения, $m_{0}-$ количество событий кода $00 . .00$.

Погрешность усиления (мультипликативная погрешность) представляет собой разность между реальной и идеальной передаточными характеристиками в точке максимального выходного значения при условии нулевой аддитивной погрешности (при отсутствии смещения) и определяется согласно формуле:

$$
E_{g}=\frac{\left(V_{r e f}-\frac{3 \mathrm{v}_{\mathrm{i}}}{2}\right)-\left(V_{1}-\frac{m_{g} * \Delta \mathrm{V}}{M}\right)}{v_{i}}-E_{0},
$$

где $V_{1}$-конечное значение входного тестового напряжения, $m_{g}$ - количество событий кода $11 . .11, V_{r e f}$ - опорное напряжение АЦП. 
Для синусоидального входного сигнала вероятность выпадения кодов не одинакова. Для $N$-разрядного АЦП с входным диапазоном полной шкалы $\pm V_{F S}$ и амплитудой $A$, вероятность появления кода $n$ определяется выражением $[6,7,8]$ :

$$
p(n)=\frac{1}{\pi} *\left(\arcsin \left(\frac{V_{F S^{*}}\left(n-2^{N-1}\right)}{A * 2^{N}}\right)-\arcsin \left(\frac{V_{F S^{*}}\left(n-1-2^{N-1}\right)}{A * 2^{N}}\right)\right) .
$$

График этой функции вместе с реальным распределением выходных кодов для микросхемы AD7888 представлен на рис.4. Стоит обратить внимание, что вероятность выпадения кода возрастает в пиках синусоиды, так как $d v / d t$ в этих точках мало (больше попаданий в интервал), чем в районе пересечения нуля, где $d v / d t$ максимально (меньше попаданий в интервал).

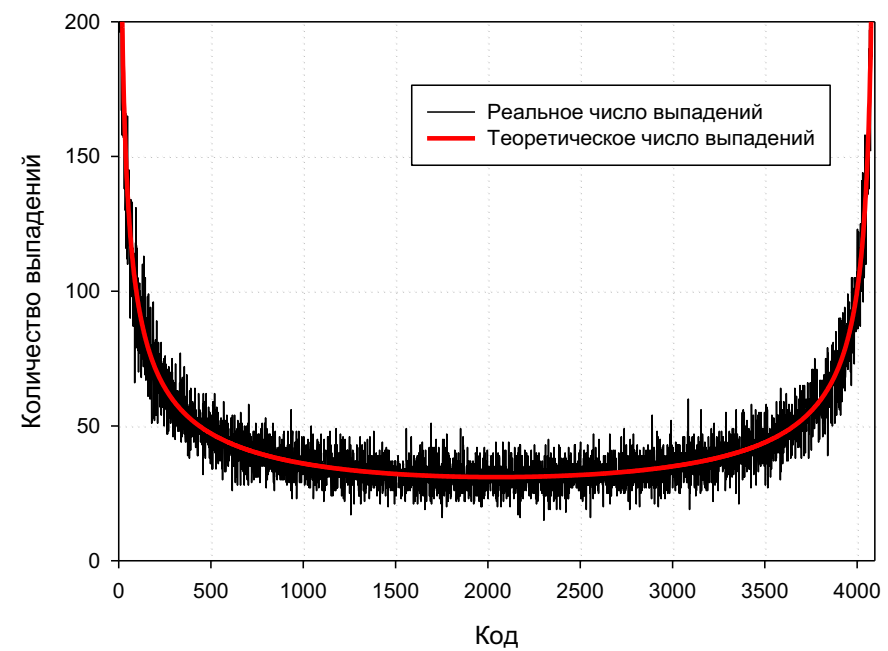

Рис. 4. Сопоставление реального распределения выходных кодов микросхемы AD7888 с теоретическим

(Fig. 4. Actual and theoretical code distribution for ADC AD7888)

Для вычисления точностных параметров АЦП из данной статистики необходимо рассчитать теоретическое число выпадений каждого кода согласно формуле:

$$
h(n)_{\text {теор. }}=p(n) * M .
$$

Соответствующая погрешность ДНЛ и ИНЛ определяются выражениями:

$$
\begin{aligned}
& \operatorname{DNL}(n)=\frac{h(n)_{\text {реал. }}}{p(n) * M}-1, \\
& \operatorname{INL}(n)=\sum_{i=1}^{n} \operatorname{DNL}(n) .
\end{aligned}
$$

Стоит отметить, что для корректного вычисления погрешности ИНЛ после вычисления кумулятивной суммы из ДНЛ необходимо построить наиболее подходящую линию, таким образом, чтобы ИНЛ равнялась 0 в конечных точках, а затем разность двух полученных зависимостей даст ИНЛ [4].

При измерении ИНЛ и ДНЛ гистограммным методом в качестве входного сигнала чаще используется синусоидальный, а не линейно возрастающий сигнал, так как при соответствующей фильтрации можно формировать синусоидальные сигналы с крайне высокой линейностью и низким шумом. На рис. 5а и 56 представлены графики зависимостей ДНЛ и ИНЛ для линейно возрастающего и синусоидального входного сигнала соответственно. 
Роман С. Торшин, Георгий С. Сорокоумов, Дмитрий В. Бобровский,

Александр А. Демидов, Анастасия В. Уланова

ИЗМЕРЕНИЕ КРИТЕРИАЛЬНЫХ ПАРАМЕТРОВ АНАЛОГО-ЦИФРОВЫХ

ПРЕОБРАЗОВАТЕЛЕЙ И КОНТРОЛЬ ИХ ИЗМЕНЕНИЯ ВО ВРЕМЯ

РАДИАЦИОННОГО ЭКСПЕРИМЕНТА

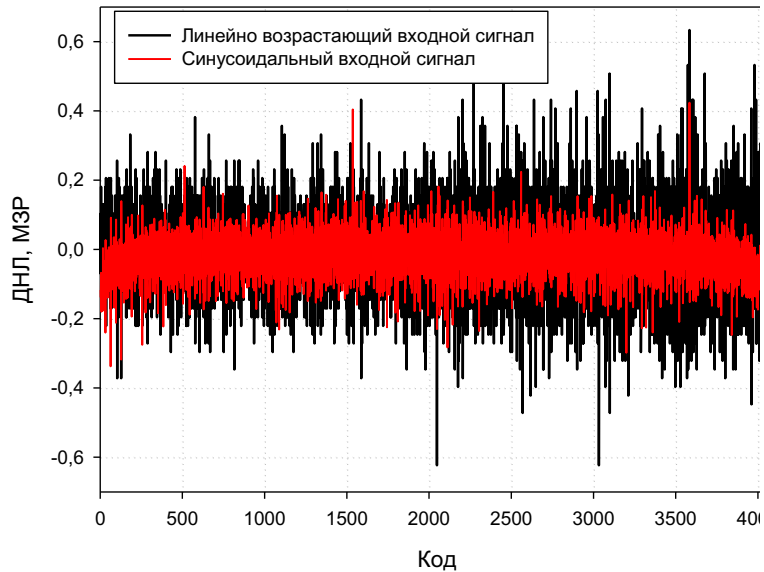

Puс. 5а. ДНЛ микросхемы АD7888 при синусоидальном и линейно возрастающем входном сигнале

(Fig. 5a. DNL for sinusoidal and linear ramp input signals for ADC AD7888)

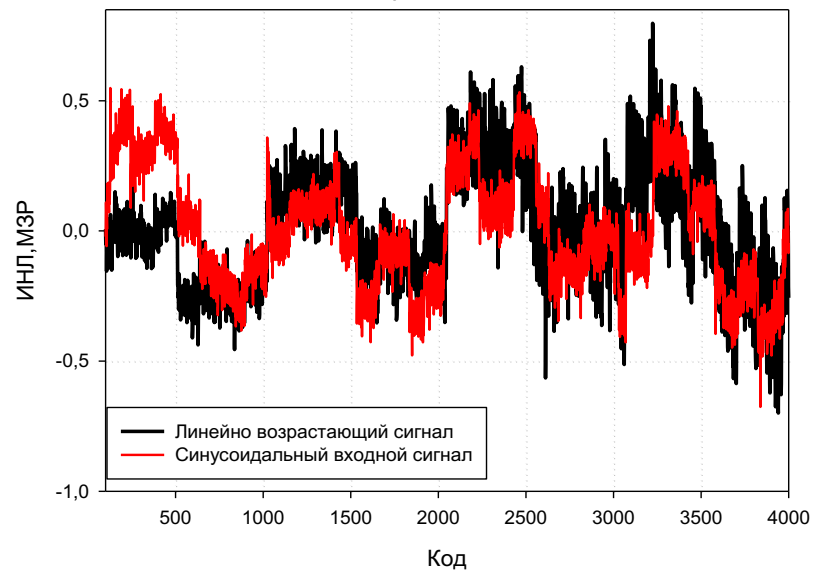

Рис. 5б. ИНЛ микросхемы АD7888 при синусоидальном и линейно возрастающем входном сигнале

(Fig. 5b. INL for sinusoidal and linear ramp input signals for $A D C$ AD7888)

Из приведенных рис. 5а и 5б видно, что использование в качестве сигнала прецизионного синуса с фильтром низких частот дает лучшие значения ДНЛ и ИНЛ, что объясняется тем, что точность измерения ИНЛ и ДНЛ АЦП не может превышать точность ИНЛ входного сигнала с ЦАП, а также любой высокочастотный шум, присутствующий в линейно изменяющимся сигнале нельзя убрать фильтрацией (поскольку она тоже повлияет на линейность).

Гистограммный тест устраняет влияние приведенного к входу шума путем усреднения его по всем кодовым интервалам. Поэтому он хорошо подходит для современных высокочастотных АЦП с широкой полосой, совместим со стандартным программным обеспечением ПК и оценочных плат производителей АЦП. Из недостатков метода - невозможность определения монотонности статической ХП АЦП, то есть порядок появления кодов по отношению к входному сигналу с помощью этого теста определить нельзя.

\section{2. Расчет динамических параметров АЦП на основе методов ЦОС}

Для определения динамических характеристик АЦП также используются методы ЦОС. Дискретное преобразование Фурье (ДПФ) над оцифрованным сигналом позволяет преобразовать его из временной области, в его эквивалентное представление в частотной. Количество отсчетов М для ДПФ должно равняться степени двойки (необходимо для процедур вычисления быстрого преобразования Фурье (БПФ) - алгоритма, позволяющего уменьшить требуемое число математических вычислений). На рис. 6 представлен усредненный результат от взятия десяти БПФ от выходного сигнала АЦП AD7888 с приведенными значениями основных динамических параметров АЦП: SNR, SINAD, THD, SFDR. Усреднение результатов нескольких отдельных БПФ не приводит к изменению шумового порога, а только уменьшает разброс шумовых составляющих.

Отношение сигнал/шум (SNR) - это отношение среднеквадратического значения величины входного сигнала к среднеквадратическому значению величины шума (за исключением гармонических искажений), выраженное в децибелах.

Отношение сигнал/шум и коэффициент искажений (SINAD, или S/N+D) - это отношение среднеквадратичного значения амплитуды сигнала к среднему значению корня 
из суммы квадратов всех других спектральных компонентов, включая гармоники, но исключая постоянную составляющую.

Динамический диапазон, свободный от гармоник (SFDR) определяется как отношение среднеквадратичного значения амплитуды сигнала к наиболее мощной паразитной гармоники в первой зоне Найквиста от 0 до $f_{s} / 2$. Обычно SFDR выражается относительно амплитуды несущей (дБн).

Полные нелинейные искажения (THD) определяются как отношение среднеквадратичного значения основной частоты сигнала к среднему значению корня из суммы квадратов гармоник (обычно существенны только первые пять) [1].

Из рис. 6 видно, что минимальный уровень шума, полученного с помощью БПФ, приблизительно равен 110 дБ от полной шкалы АЦП, тогда как теоретическое отношение сигнал/шум 12-разрядного АЦП равно 74 дБ. Минимальный уровень шума от БПФ не равен отношению сигнал/шум АЦП, потому что БПФ действует, подобно аналоговому анализатору спектра с шириной полосы $\frac{f_{S}}{M}[1]$.

Теоретически минимальный уровень шума БПФ равен $10 \log \left(\frac{M}{2}\right)$ дБ, то есть ниже минимального уровня шума квантования из-за выигрыша БПФ в отношении сигнал/шум [2, 4]. При испытаниях АЦП, использующих БПФ, важно быть уверенным, что количество точек БПФ достаточно велико для того, чтобы нелинейные искажения можно было отличить от минимального уровня шума БПФ.

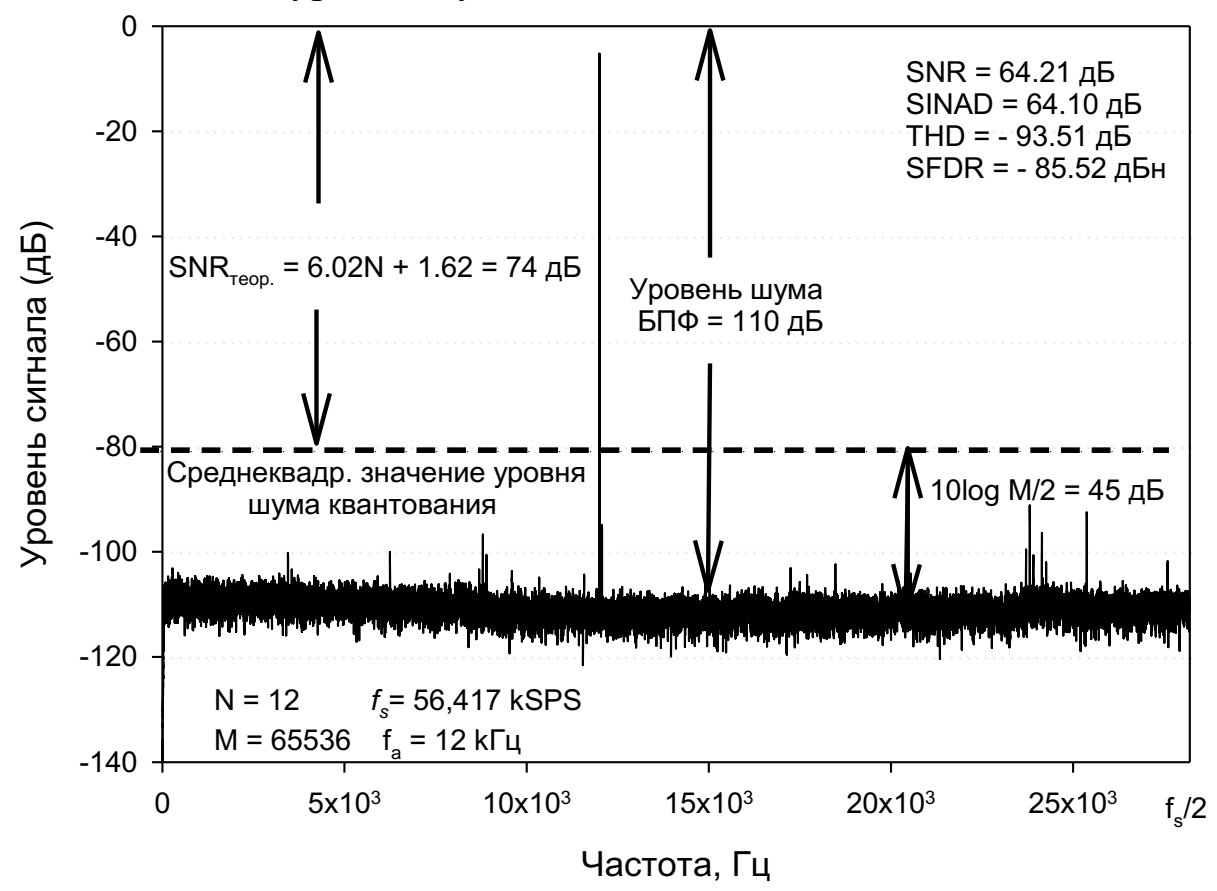

Рис. 6. БПФ массива 65536 точек для микросхемы АЦП АD7888 (Fig. 6. FFT array of 65536 points for ADC AD7888)

\section{3. Используемое оборудование}

Для измерения критериальных параметров микросхемы AD7888 использовался следующий набор модулей, объединенных в единую контрольно-измерительную систему при помощи NI PXI-1033 - PXI шасси со встроенным дистанционным контроллером:

NI PXI-4461 - звуковой и вибрационный модуль, располагающий двумя каналами для динамической генерации сигнала и двумя каналами для оцифровки. Использовался в качестве генератора линейно возрастающего сигнала (24-разрядный ЦАП). 
NI PXI-7841R - многофункциональный модуль PXI, имеющий в своем составе ПЛИC Virtex-5 LX30, 96 цифровых линий, 8 аналоговых входов и 8 аналоговых выходов с 16-битным разрешением.

NI PXI-4110 - 3-х канальный программируемый источник питания.

NI PXI-4071 - высокопроизводительный цифровой мультиметр с разрешением $71 / 2$ разряда и напряжением $1000 \mathrm{~B}$.

Для подачи синусоидального сигнала использовался SMA100B - генератор сигналов, предназначенный для формирования немодулированных колебаний высокой спектральной чистоты в диапазоне от 8 кГц до 3 ГГц.

ПО для связи всех используемых блоков и проведения автоматизированного радиационного эксперимента было написано в CAПP LabVIEW.

\section{4. Проведение автоматизированного радиационного эксперимента}

Исследования на стойкость микросхемы AD7888 к воздействию накопленной дозы проводились с использованием рентгеновского источника «РИК-0401».

Алгоритм обмера критериальных параметров исследуемой микросхемы при работе на рентгеновском источнике был следующим: каждые 30 с проводился ФК микросхемы, и параметры АЦП (ИНЛ, ДНЛ, SNR, SINAD, $V_{r e f,} I_{c c}$ ) записывались в файл-отчет, на анализе которого в последствии была установлена их деградация из-за воздействия дозового эффекта $[9,10]$.

Типичными эффектами от поглощенной дозы в АЦП является деградация ХП микросхемы и связанные с этим ухудшения точностных параметров: интегральной нелинейности, дифференциальной нелинейности, ошибки смещения и усиления $[11,12]$. График изменения ХП АЦП при воздействии поглощенной дозы для разных значений поглощенной дозы приведен на рис. 7.

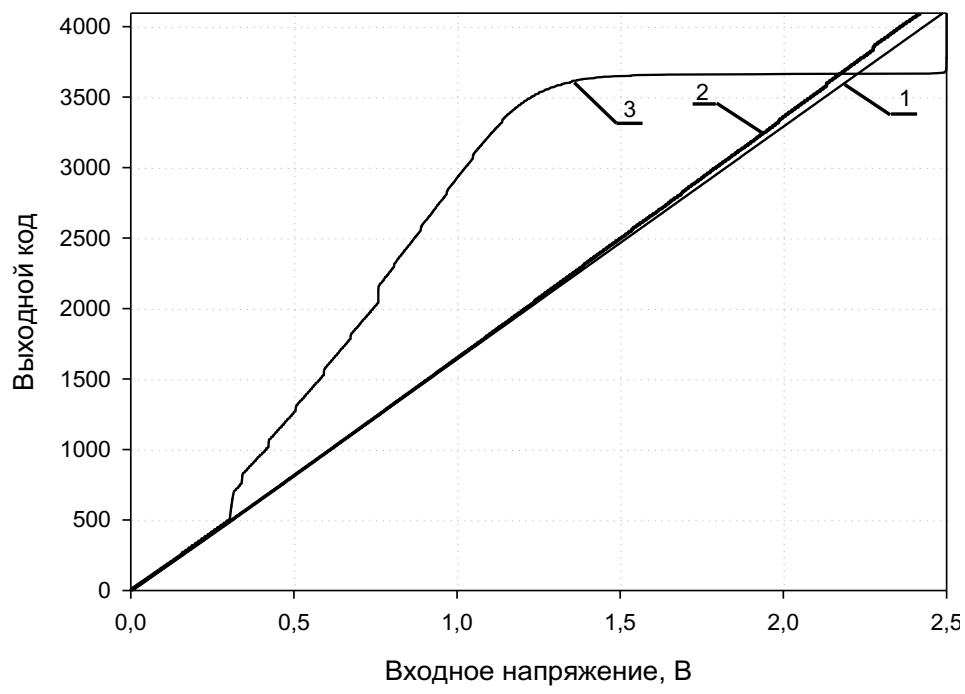

Рис. 7. ХП АЦП для различных уровней набранной дозы:

1 - исходная ХП; $2-9 \times 10^{3}$ ед.; $3-11 \times 10^{3}$ ед.

(Fig. 7. ADC transfer function for different irradiation steps

1 - initial TF; $2-9 \times 10^{3}$ un.;3 $-11 \times 10^{3}$ un.)

Графики изменения электрических параметров (тока потребления и напряжения внутреннего источника опорного напряжения (ИОН)) приведены на рис. 8а и $8 б$ соответственно. Образец №4 облучался в режиме работы с внешним ИОН. 
Роман С. Торшин, Георгий С. Сорокоумов, Дмитрий В. Бобровский,

Александр А. Демидов, Анастасия В. Уланова

ИЗМЕРЕНИЕ КРИТЕРИАЛЬНЫХ ПАРАМЕТРОВ АНАЛОГО-ЦИФРОВЫХ

ПРЕОБРАЗОВАТЕЛЕЙ И КОНТРОЛЬ ИХ ИЗМЕНЕНИЯ ВО ВРЕМЯ

РАДИАЦИОННОГО ЭКСПЕРИМЕНТА

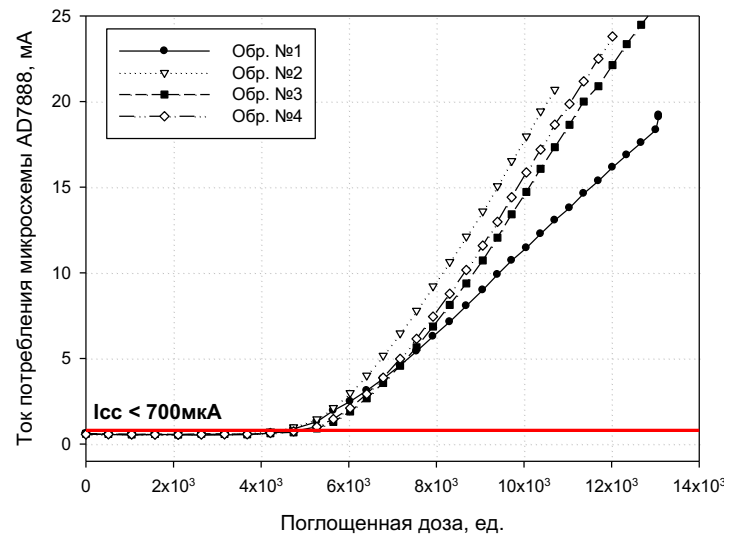

Рис. 8а. Изменение тока потребления микросхемы AD7888 при воздействии поглощенной дозы

(Fig. 8a. Power supply currents AD7888 during irradiation)

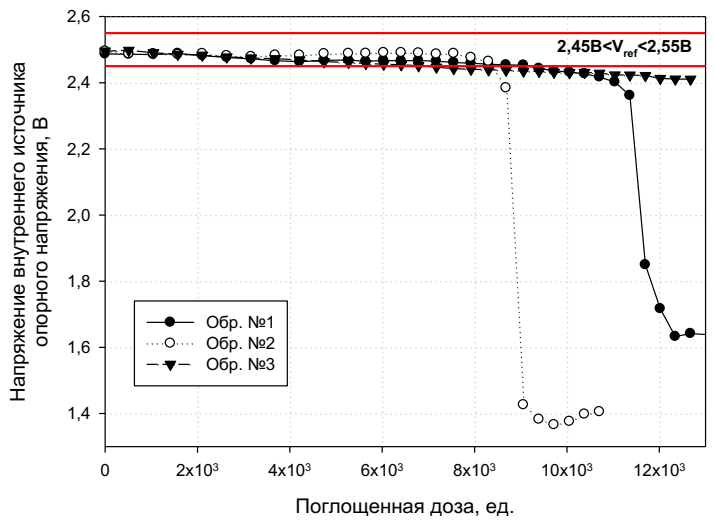

Рис. 8б. Изменение напряжения внутреннего ИОН микросхемы АD7888 при воздействии поглощенной дозы

(Fig. 8b. The input reference voltage AD7888 during irradiation)

График изменения ДНЛ на образце №1 в зависимости от поглощенной дозы, а также график изменения минимального и максимального ДНЛ приведены на рис. 9а и 9б соответственно. Видно, что АЦП начинает пропускать коды, начиная примерно с $8 \times 10^{3}$ ед. Выход параметра за указанные в документации нормы обозначен красной линией на графике.

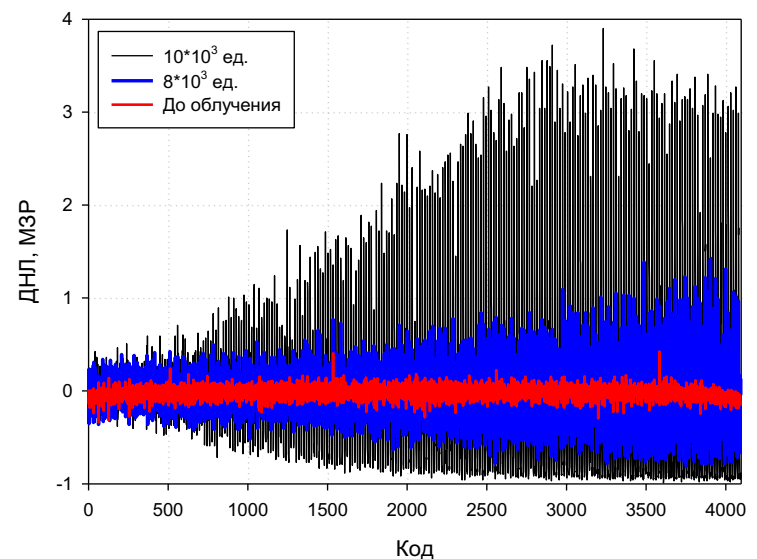

Рис. 9а. Изменение ДНЛ микросхемы АD7888 при воздействии поглощенной дозы

(Fig .9a. DNL AD7888 for different irradiation steps)

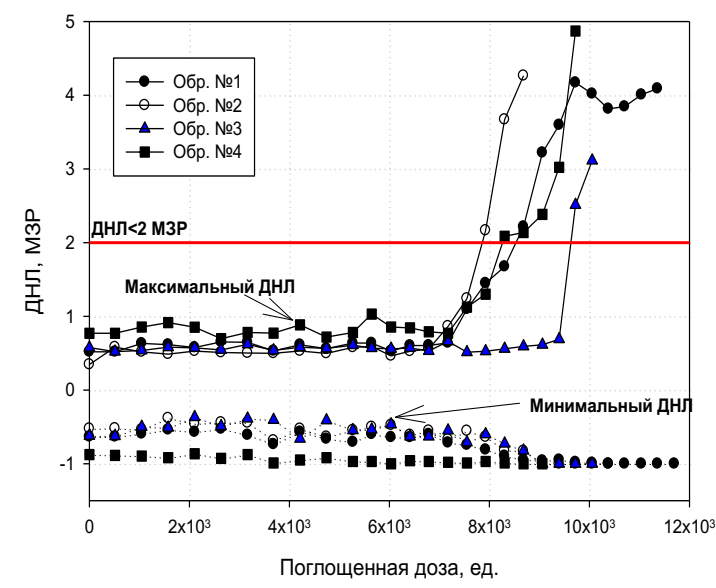

Рис. 9б. Изменение максимальной и минимальной ДНЛ микросхемы АD7888 при воздействии поглощенной дозы

(Fig. 9b. DNL AD7888 during irradiation)

ИНЛ является мерой отклонения фактической ХП от идеальной. Видно, что реальная ХП все дальше отклоняется от идеальной в обе стороны. Это можно увидеть на рис. 10а, 106 . 
Роман С. Торшин, Георгий С. Сорокоумов, Дмитрий В. Бобровский, Александр А. Демидов, Анастасия В. Уланова

ИЗМЕРЕНИЕ КРИТЕРИАЛЬНЫХ ПАРАМЕТРОВ АНАЛОГО-ЦИФРОВЫХ

ПРЕОБРАЗОВАТЕЛЕЙ И КОНТРОЛЬ ИХ ИЗМЕНЕНИЯ ВО ВРЕМЯ

РАДИАЦИОННОГО ЭКСПЕРИМЕНТА

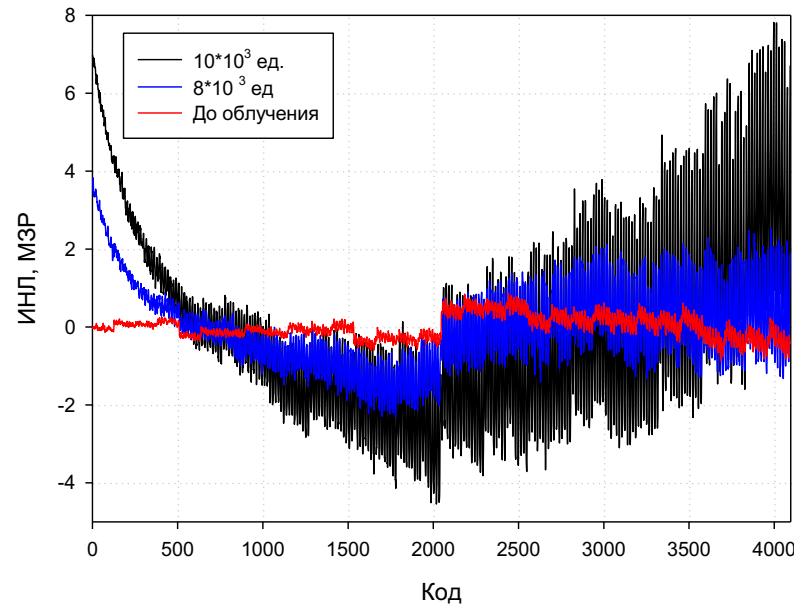

Рис. 10а. Изменение ИНЛ микросхемы АD7888 при воздействии

поглощуенной дозы

(Fig. 10a. INL AD7888 for different irradiation steps)

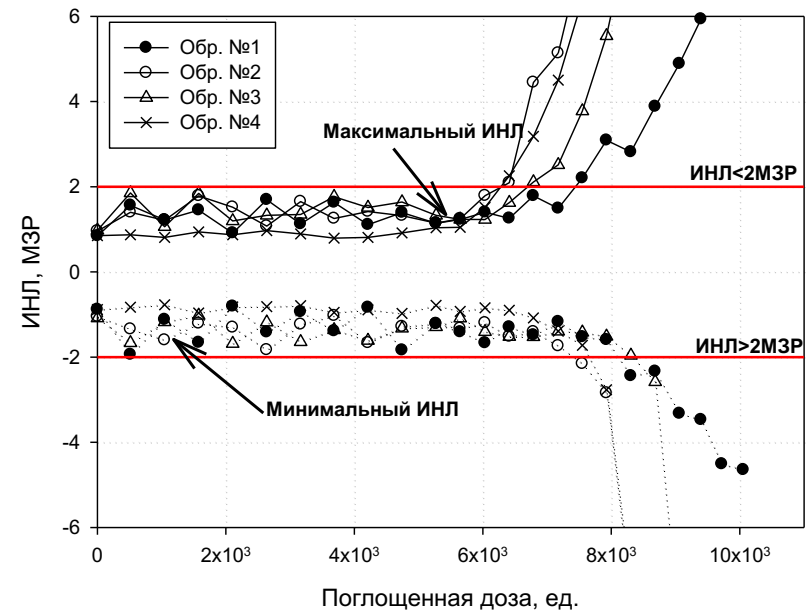

Рис. 10б. Изменение максимальной и минимальной ИНЛ микросхемы АD7888 при воздействии поглощенной дозы

(Fig. 10b. INL AD7888 during irradiation)

Можно отметить различное поведение графиков ИНЛ и ДНЛ при накоплении дозы. На графике ИНЛ наблюдается рост «зубьев» и увеличивается общее искажение (изгиб), в то же время на графике ДНЛ наблюдается увеличение амплитуды пиков при определенных выходных кодах АЦП [3].

Изменение ошибки усиления АЦП AD7888 представлено на рис. 11. Видно, что начиная с $8 \times 10^{3}$ ед. ошибка усиления начинает сильно увеличиваться. Это можно хорошо наблюдать на графике изменения ХП АЦП AD7888 в зависимости от поглощенной дозы (рис. 7).

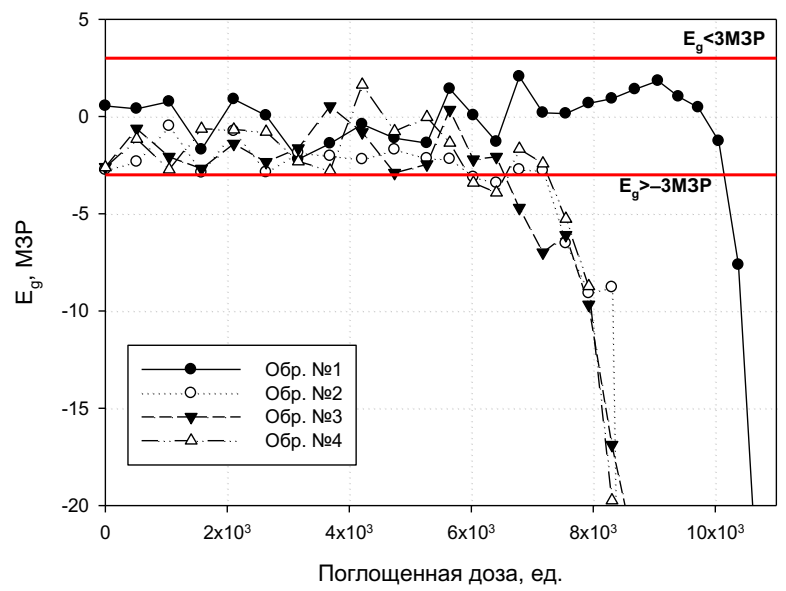

Рис. 11. Изменение ошибки усиления АЦП АD7888 при воздействии поглощенной дозы (Fig. 11. ADC AD7888 gain error during irradiation) 
Роман С. Торшин, Георгий С. Сорокоумов, Дмитрий В. Бобровский, Александр А. Демидов, Анастасия В. Уланова

ИЗМЕРЕНИЕ КРИТЕРИАЛЬНЫХ ПАРАМЕТРОВ АНАЛОГО-ЦИФРОВЫХ

ПРЕОБРАЗОВАТЕЛЕЙ И КОНТРОЛЬ ИХ ИЗМЕНЕНИЯ ВО ВРЕМЯ

РАДИАЦИОННОГО ЭКСПЕРИМЕНТА

Изменение динамических параметров SNR и SINAD приведено на рис. 12а и 126.

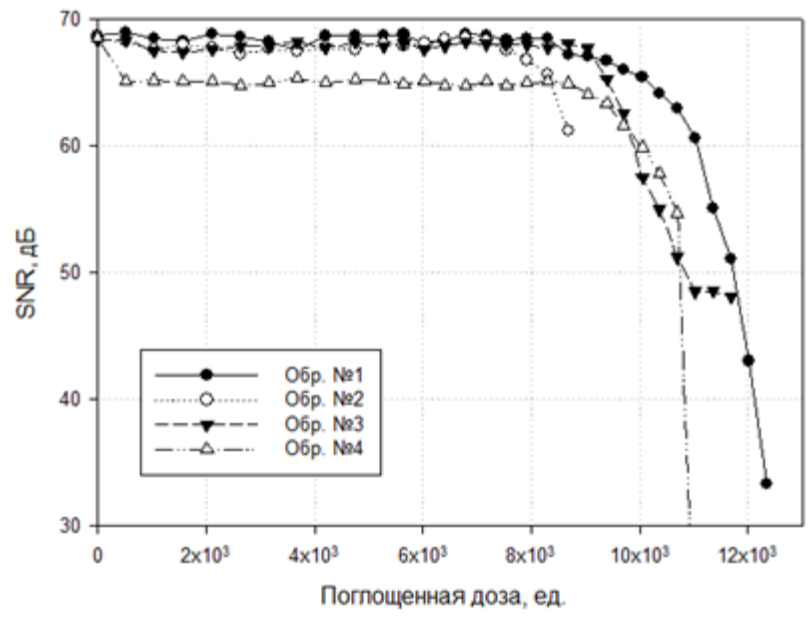

Puc. 12a. Изменение SNR микросхемы AD7888 при воздействии поглощенной дозы

(Fig. 12a. ADC AD7888 SNR during irradiation)

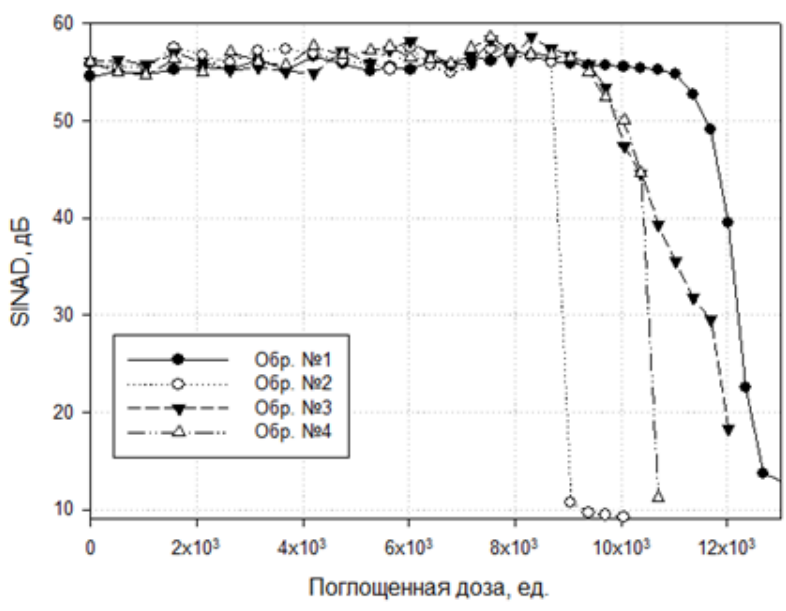

Puc. 126. Изменение SINAD микросхемы AD7888 при воздействии поглощенной дозы

(Fig. 12b. ADC AD7888 SINAD during irradiation)

Результат выполнения БПФ над выходным оцифрованным сигналом АЦП AD7888 до облучения и после набора $12 \times 10^{3}$ ед. представлен на рис. 13. Частота выборки $f_{s}=104 \mathrm{kSPS}$, частота сигнала $f_{a}=10$ кГц, количество отсчетов БПФ $=65536$ точек.

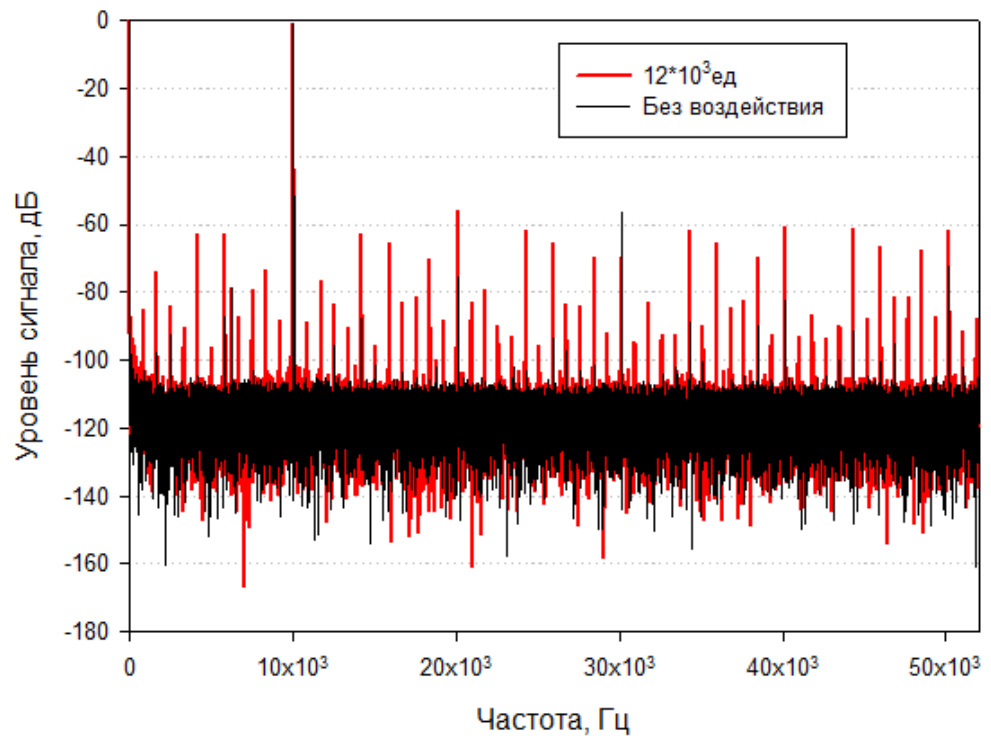

Рис. 13. Результат выполнения БПФ над выходным оичированным сигналом АЦП АD7888 до облучения и после набора $12 \times 10^{3}$ ед.

(Fig. 13. FFT array of 65536 points for ADC AD7888 before and after irradiation)

\section{Заключение}

Представленные методы тестирования динамических и статических параметров АЦП позволяют дать полную картину об испытываемой микросхеме, а в совокупности с установками, имитирующими воздействия ионизирующего излучения (ИИ) на интегральные схемы (ИС), позволяют прогнозировать их поведение в условиях воздействия 
Роман С. Торшин, Георгий С. Сорокоумов, Дмитрий В. Бобровский,

Александр А. Демидов, Анастасия В. Уланова

ИЗМЕРЕНИЕ КРИТЕРИАЛЬНЫХ ПАРАМЕТРОВ АНАЛОГО-ЦИФРОВЫХ

ПРЕОБРАЗОВАТЕЛЕЙ И КОНТРОЛЬ ИХ ИЗМЕНЕНИЯ ВО ВРЕМЯ

РАДИАЦИОННОГО ЭКСПЕРИМЕНТА

ИИ космического пространства. Данные методы могут быть реализуемы на одном оборудовании, что характеризует их универсальность.

Необходимость полноценного измерения всех критериальных параметров обуславливается в дальнейшем применении микросхем [8]: к примеру, есть приложения, в которых АЦП используется для измерения медленно изменяющихся сигналов. В таком случае статические погрешности будут играть главную роль, а при использовании АЦП в таких приложениях как видео и связь важны высокие значения SFDR и SNR.

\section{СПИСОК ЛИТЕРАТУРЫ:}

1. Кестер У. Аналого-цифровое преобразование. - М.: Техносфера, 2007. - 1016 с. ISBN 978-5-94836-146-8.

2. W. Kester, "A Brief History of Data Conversion: A Tale of Nozzles, Relays, Tubes, Transistors, and CMOS," in IEEE Solid-State Circuits Magazine. Vol. 7, no. 3. P. 16-37, Summer 2015. DOI: https://doi.org/10.1109/MSSC.2015.2442371.

3. Торшин Р.С. / Проявление сбоев при воздействии ТЗЧ в современном ЦАП / Сорокоумов Г.С., Бобровский Д.В., Демидов А.А., Калашников О.А., Телец В.А. // 22-я Всероссийская научно-техническая конференция «Стойкость-2019»: тезисы докладов. 2019, Лыткарино, 4-5 июня. С. 244-245.

4. Kester W. / Mixed-Signal and DSP Design Techniques. - M.: Newnes/Elsevier, 2002. - 611 c.

5. I.O. Loskutov, A.B. Karakozov and P.V. Nekrasov, "Automated test setup for functional and parametrical control of microcontrollers with internal ADC," 2016 International Siberian Conference on Control and Communications (SIBCON), Moscow, 2016. P. 1-4. DOI: https://doi.org/10.1109/SIBCON.2016.7491799.

6. IEEE Standard for Terminology and Test Methods for Analog-to-Digital Converters," in IEEE Std 1241-2010 (Revision of IEEE Std 1241-2000). P. $1-139, \quad 14 \quad$ Jan. 2011 , DOI: https://doi.org/10.1109/IEEESTD.2011.5692956.

7. A.O. Akhmetov et al., "Proton Accelerator's Direct Ionization Single Event Upset Test Procedure," 2019 IEEE 31st International Conference on Microelectronics (MIEL), Nis, Serbia, 2019. P. 107-110, DOI: https://doi.org/10.1109/MIEL.2019.8889634.

8. A.Y. Egorov, I.A. Mozhaev, P.V. Nekrasov, D.V. Boychenko and I.O. Loskutov, "Comparison of on-chip ADC testing techniques," 2017 IEEE 30th International Conference on Microelectronics (MIEL), Nis, 2017. P. 247250. DOI: https://doi.org/10.1109/MIEL.2017.8190113.

9. Барбашов, Вячеслав М.; Калашников, Олег А. Функционально-логическое моделирование дозовых радиационных отказов сф-блоков систем на кристалле. Безопасность информационных технологий, [S.1.]. T. 24, № 4. P. $40-86, \quad$ ноябрь $2017 . \quad$ ISSN $2074-7136$. URL: $\quad$ https://bit.mephi.ru/index.php/bit/article/view/283 (дата обращения: 20.07.2020). DOI: http://dx.doi.org/10.26583/bit.2017.4.09.

10. Чумаков А.И. и др. Оценка показателей стойкости интегральных схем при воздействии тяжелых заряженных частиц с использованием различных моделей. Безопасность информационных технологий, [S.1.]. T. 24, № 1. C. 73-84, 2017. ISSN 2074-7136. URL: https://bit.mephi.ru/index.php/bit/article/view/58. DOI: http://dx.doi.org/10.26583/bit.2017.1.09.

11. Никифоров А.Ю. / Радиационные эффекты в КМОП ИС.//Телец В.А., Чумаков А.И. - М.: Радио и связь, 1994. - $165 \mathrm{c}$.

12. Чумаков А.И. / Действие космической радиации на интегральные схемы. - М. - Радио и связь, 2004. $319 \mathrm{c}$.

13. Dressendorfer P.V. / Ionizing Radiation Effects in MOS devices and Circuits, // T.P.Ma. - M.: Willey, 1989. $587 \mathrm{p}$.

14. Demidov, Alexander \& Kalashnikov, Oleg \& Nikiforov, A. \& Tararaksin, Alexander \& Telets, Vitaly. (2015). Radiation Behavior and Test Specifics of A-D and D-A Converters. Informacije MIDEM. 45. 153-159.

15. A.B. Karakozov, P.V. Nekraso, D.V. Bobrovsky, G.S. Sorokoumov and V.A. Telets, "Single Event Effects And Total Dose Testing Of Digital To Analog Converters," 2017 17th European Conference on Radiation and Its Effects on Components and Systems (RADECS), Geneva, Switzerland, 2017. P. 1-5, DOI: https://doi.org/10.1109/RADECS.2017.8696263.

\section{REFERENCES:}

[1] Kester W. Analog-Digital Conversion. - Copyright (C) 2004 By Analog Devices, Inc. Printed in the United States of America., 2007. - 1016 p.

[2] Kester W. "A Brief History of Data Conversion: A Tale of Nozzles, Relays, Tubes, Transistors, and CMOS," in IEEE Solid-State Circuits Magazine. Vol. 7, no. 3. P. 16-37, Summer 2015. DOI: https://doi.org/10.1109/MSSC.2015.2442371. 
Роман С. Торшин, Георгий С. Сорокоумов, Дмитрий В. Бобровский,

Александр А. Демидов, Анастасия В. Уланова

ИЗМЕРЕНИЕ КРИТЕРИАЛЬНЫХ ПАРАМЕТРОВ АНАЛОГО-ЦИФРОВЫХ

ПРЕОБРАЗОВАТЕЛЕЙ И КОНТРОЛЬ ИХ ИЗМЕНЕНИЯ ВО ВРЕМЯ

РАДИАЦИОННОГО ЭКСПЕРИМЕНТА

[3] Torshin R.S. Manifestation of failures under the influence of HCP in a modern DAC. Sorokoumav G.S., Bobrovsky D.V., Demidov A.A., Kalashnikov O.A., Telets V.A. 22-ya Vserossiyskaya nauchno-tehnicheskaya konferenciya «Stoykost'-2019»: tezisi dokladov. 2019, Litkarino, 4-5 ijynya. S. 244-245. (in Russian).

[4] Kester W. Mixed-Signal and DSP Design Techniques. - M.: Newnes/Elsevier, 2002. - 611 p.

[5] I.O. Loskutov, A.B. Karakozov and P.V. Nekrasov, "Automated test setup for functional and parametrical control of microcontrollers with internal ADC," 2016 International Siberian Conference on Control and Communications (SIBCON), Moscow, 2016. P. 1-4. DOI: https://doi.org/10.1109/SIBCON.2016.7491799.

[6] IEEE Standard for Terminology and Test Methods for Analog-to-Digital Converters," in IEEE Std 1241-2010 (Revision of IEEE Std 1241-2000). P. $\quad 1-139, \quad 14 \quad$ Jan. 2011 , DOI: https://doi.org/10.1109/IEEESTD.2011.5692956.

[7] A.O. Akhmetov et al., "Proton Accelerator's Direct Ionization Single Event Upset Test Procedure," 2019 IEEE 31st International Conference on Microelectronics (MIEL), Nis, Serbia, 2019. P. 107-110, DOI: https://doi.org/10.1109/MIEL.2019.8889634.

[8] A.Y. Egorov, I.A. Mozhaev, P.V. Nekrasov, D.V. Boychenko and I.O. Loskutov, "Comparison of on-chip ADC testing techniques," 2017 IEEE 30th International Conference on Microelectronics (MIEL), Nis, 2017. P. 247-250. DOI: https://doi.org/10.1109/MIEL.2017.8190113. Barbashov, Vyacheslav M.; Kalashnikov, Oleg A. Functional-logic simulation of IP-blocks dose functional

[9] Barbashov, Vyacheslav M.; Kalashnikov, Oleg A. Functional-logic simulation of IP-blocks dose functional failures. IT Security (Russia), [S.1.]. V. 24, no. 4. P. 80-86, nov. 2017. ISSN 2074-7136. URL: $\quad$ https://bit.mephi.ru/index.php/bit/article/view/283 20.07.2020). DOI: http://dx.doi.org/10.26583/bit.2017.4.09.

[10] Chumakov A.I. et al. Evaluation of indicators of resistance of integrated circuits when exposed to heavy charged particles using various models. IT Security (Russia), [S.1.]. V. 24, no 1. P. 73-84, 2017. ISSN 2074-7136. URL: https://bit.mephi.ru/index.php/bit/article/view/58. DOI: http://dx.doi.org/10.26583/bit.2017.1.09.

[11] Nikiforov A.Yu. Radiation effects in CMOS IC . Telets V.A., Chumakov A.I. - M.: Radio and communications, 1994. - 165 p. (in Russian).

[12] Chumakov A.I., Effects of Cosmic Radiation on IC, Moscow: Radio i Svyaz', 2004. - 319 p. (in Russian).

[13] Dressendorfer P.V. Ionizing Radiation Effects in MOS devices and Circuits, T.P.Ma. - M.: Willey, 1989. - 587 p.

[14] Demidov, Alexander \& Kalashnikov, Oleg \& Nikiforov, A. \& Tararaksin, Alexander \& Telets, Vitaly. (2015). Radiation Behavior and Test Specifics of A-D and D-A Converters. Informacije MIDEM. 45. 153-159.

[15] A.B. Karakozov, P.V. Nekraso, D.V. Bobrovsky, G.S. Sorokoumov and V.A. Telets, "Single Event Effects And Total Dose Testing Of Digital To Analog Converters," 2017 17th European Conference on Radiation and Its Effects on Components and Systems (RADECS), Geneva, Switzerland, 2017. P. 1-5, DOI: https://doi.org/10.1109/RADECS.2017.8696263.

Поступила в редакиию - 26 июля 2020 г. Окончательный вариант - 20 августа 2020 г. Received - July 26, 2020. The final version-August 20, 2020. 